\title{
Digital teaching files - a useful teaching tool for the modern radiologist
}

\section{André Tertius du Plessis, MB ChB, MMed Rad Diag, FC Rad Diag (SA) \\ Department of Radiology, Steve Biko Academic Hospital, Pretoria (current affiliations: Scholtz and Partners, and Department of Radiology, University of Pretoria)}

\section{Introduction}

Radiologists have always collected copies of model examples and interesting cases encountered in daily practice to use for teaching purposes. ${ }^{1,2}$ A collection of teaching files is an important resource for medical education and the dissemination of knowledge in radiology. Furthermore, the presence of a radiological teaching file is also a requirement at several universities and tertiary institutions in South Africa and abroad, where radiology postgraduate training is conducted. $^{2}$

The advent of digital radiology and the more widespread use of picture archiving and communication systems (PACS) have led to a unique opportunity to change the way that radiology teaching cases are collected, stored and managed. Traditional teaching file systems are rapidly becoming obsolete and incompatible with the digital environment of modern radiology departments. ${ }^{3,4}$

\section{Traditional v. digital teaching files}

The traditional way in which radiology teaching cases were collected comprised mostly film-based teaching file systems that were compiled and stored in a store room or filing cabinet. The cases would typically be stored or archived according to anatomical site or pathological process, often using the American College of Radiology (ACR) anatomical or pathological codes as indices.

With the advent of digital radiology, radiological images are acquired in an inherent digital format, which lends itself to the unique position of digital teaching files (DTF). With a DTF, the selected images and appropriate information are stored electronically and then recalled for review on a personal computer.

\section{Shortcomings of traditional teaching files}

Compilation of film-based teaching cases was cumbersome, with several steps needed to create a teaching case:

- identify an appropriate case

- request copies of the selected radiographs

- write clinical and radiological information on the film packet

- code according to the filing system

- file in store room or cabinet.

Managing and storing traditional teaching file cases can also be problematic, especially if the library consists of hundreds or thousands of cases. Not only is accurate archiving of the cases time-consuming but physical storage space can also be a problem. The additional issue of film degradation, when film is kept over long periods (especially if not under optimum conditions), can also be problematic. Radiograph envelope degradation is also of concern.

Cases can only be viewed at one location at a specific time - this may therefore limit the availability of cases if there are multiple viewers at different locations.

Duplication of traditional teaching cases to share them among different sites is possible although less than ideal - radiographs need to be duplicated, with resultant loss in image quality.

\section{Characteristics of the ideal DTF}

The creation of a DTF requires several steps. First, a suitable case has to be selected and appropriate selected images identified. The selected images should then be submitted in the appropriate format to the teaching archive. The case should then be edited to include annotations, relevant clinical history, teaching notes or tips, and references (if available) to optimise its teaching potential. Once the case has been completed, it should be made available for use or review by the targeted users. $^{1}$

The ideal DTF system should therefore possess the following characteristics to maximise its usefulness:

- Versatility. The system should be able to support the whole process of case preparation from image acquisition through image editing and case annotation, to structured organisation of cases, comprehensive search and cross-referencing functionality (on multiple parameters), and secure image storage. ${ }^{1}$

- Accessibility. DTF cases should be easily accessible either within the hospital computer network or internet (if desired). Selective access rights may also be implemented to prevent unauthorised access or inappropriate use. The ideal system should employ some form of copyright protection to prevent unauthorised duplication.

- Ease of use. The system should be user-friendly, quick to use and intuitive. Where possible, integration into the daily workflow of the PACS system is very important to minimise the impact on clinical workflow. ${ }^{1}$

- Compatibility. DTF system software and hardware should be compatible with a variety of image formats and should also seamlessly interface with departmental hardware (and software/PACS) to allow direct transfer of images and DTF case creation. ${ }^{1}$ Compatibility should be PACS vendor-independent and ideally the interface should be built on the IHE TCE (Integrating the Healthcare Enterprise Teaching File and Clinical Trial Export) protocol.

- Flexibility. The case database should be easily viewed either as unknowns or by user-defined searches. ${ }^{1}$ This would enable the system to be used as a reference library or for exam preparation if unknown cases are to be reviewed. 


\section{REVIEW ARTICLE}

- Installation. Installation should be uncomplicated, with the minimum amount of software installation needed. Web interface software is ideal in this situation, minimising software installation on the user PC.

\section{Advantages of DTF v. traditional image libraries}

Several advantages of DTF systems are apparent; ${ }^{3}$ the most prominent are:

- Cost-effectiveness. This important advantage should not be overlooked. While the initial set-up costs of DTF systems may be higher compared with the traditional system (owing to the cost of expensive hardware), the everyday maintenance and cost per case produced is less with a DTF system. The current cost of radiographic film (approximately R5 - R30 per film depending on size and type of film) adds an additional cost to each traditional teaching case, whereas DTF systems utilise electronic storage, which is significantly cheaper on a per-case basis. ${ }^{5}$ The initial cost outset may also be lessened by the fact that DTF system hardware may be utilised for different tasks.

- Simultaneous access. Owing to the digital format of DTF cases, a specific case from the DTF library can be reviewed at multiple separate locations.

- Data duplication. The ability to duplicate DTF cases very easily without any loss of image quality is also a major advantage. Therefore, potential library duplication or shared libraries between several institutions are real and viable options.

- Transfer of DTF. Transfer of a digital collection is extremely easy compared with a traditional system. This can be achieved either via network transfer or via portable storage media (e.g. DVD or portable hard disk). ${ }^{6}$

- Interactivity. With a digital system, it is possible to add interactive elements to DTF cases (i.e. self-evaluation tests or quizzes). ${ }^{7}$

- Searchability. Searches across multi-level parameters are possible (i.e. not only searching for a specific anatomical site or pathological process, but also combining anatomical site, pathological process, image modality, patient parameters and more).

- Facilitation of case creation. A digital system greatly facilitates the process of case creation compared with the traditional system. As mentioned earlier, the process of creating a traditional teaching case is cumbersome. Creating a DTF case simplifies these cumbersome steps into easier and more streamlined case creation - the selected images are transferred directly from the $\mathrm{PACS}^{8}$ to the DTF system, the user then adds information and annotations as needed via the DTF software, and the case is immediately available on the DTF library. ${ }^{9}$

\section{Current problems with DTF systems}

With the great technical advances in digital radiology and PACS, it is unfortunate that DTF systems have not received the same great level of advancement. It would seem that most PACS vendors initially underestimated the value of a comprehensive incorporated DTF system as most of the PACS software lacks incorporated DTF functionality as required. Some PACSs do, however, offer limited DTF capability, albeit with limited functionality. ${ }^{1,210-12}$ Another disadvantage of such systems is the limitation of only being able to access such cases via a PACS workstation (as such cases remain on the PACS). Additionally, this may incur separate costs, depending on the licensing model of the PACS or RIS vendor.

This drawback has led to many institutions now relying on thirdparty applications or even custom-developed DTF systems. ${ }^{3}$ The use of third-party applications has its own set of problems. Firstly, considerable effort, financial commitment and expertise are needed to implement such a DTF system, especially at larger institutions.1 Secondly, the different types of DTF solutions unfortunately do not all demonstrate inter-compatibility and functionality - this is problematic where sharing or duplication of DTF libraries is planned. This lack of inter-compatibility is also a problem when planning to move from one DTF system to another - the older DTF cases may not be compatible with the new system, leading to extra time, effort and cost to convert older cases.

Even though some DTF systems comply with most of the required functionality, integration into the normal workflow is still problematic with most systems. This unfortunately leads to under-utilisation of the DTF system, as creating cases is too time-consuming and disruptive to normal daily workflow - in such instances, case creation (and therefore educational opportunities) will take a back seat to the pressures of clinical workflow. ${ }^{2}$

Some DTF systems, on the other hand, offer the required functionality and versatility but lack the user-friendliness and ease-of-use, which unfortunately will not encourage individuals (especially those who are not computer literate) to both contribute cases and also to choose to use the teaching file system as part of their preferred learning resource. A system understandable only by computer scientists is likely to be quietly ignored.

\section{Current different types of DTF system}

There are several different third-party applications available to use as DTF system solutions. Broadly, these applications can be categorised as institution-based technology or internet-based image libraries (although there are some applications that span both groups).

\section{Institution-based technology}

The easiest and most cost-effective way to establishing a DTF system is to use a system incorporated into the PACS. Although many commercially available PACSs allow for creation of a teaching folder, these folders unfortunately often lack the desired functionality and versatility. ${ }^{2,10-12}$

There are multiple teaching file authoring packages available which have been specifically developed for radiologists. ${ }^{13-15}$ These packages are designed for rendering teaching file creation on a local network or intranet. Examples of such packages include MyPACS.net Custom (Vivalog Technologies, www.mypacs.net) and Casimage (pubimage. hcuge.ch). Casimage is a database system developed at the Digital Imaging Unit of Geneva University Hospital, Switzerland. Images can be sent directly from PACS to the DTF server and can either be converted to a joint photographic expert group (JPEG) file at the workstation or sent in digital imaging and communications in medicine (DICOM) form to the server, where they are automatically converted. Unfortunately, all these solutions come at a considerable cost: Casimage software currently 


\section{REVIEW ARTICLE}

\section{Table I. Quick reference table}

\begin{tabular}{|c|c|c|c|c|c|}
\hline & $\begin{array}{l}\text { Internet based: } \\
\text { MyPACS.net }\end{array}$ & $\begin{array}{l}\text { Hybrid: } \\
\text { MIRC }\end{array}$ & $\begin{array}{l}\text { Hybrid: } \\
\text { MyPacs.net }\end{array}$ & $\begin{array}{l}\text { Institutional: } \\
\text { Vendor based }\end{array}$ & $\begin{array}{l}\text { Institutional: } \\
\text { Casimage }\end{array}$ \\
\hline $\begin{array}{l}\text { Easy integration with } \\
\text { workflow }\end{array}$ & No & $\begin{array}{l}\text { Requires programming, } \\
\text { unless vendor uses IHE } \\
\text { TCE }\end{array}$ & $\begin{array}{l}\text { Requires } \\
\text { programming }\end{array}$ & Yes & $\begin{array}{l}\text { Requires } \\
\text { programming }\end{array}$ \\
\hline $\begin{array}{l}\text { Adding DICOM } \\
\text { images to DTF }\end{array}$ & Yes & Yes & Yes & Yes & Yes \\
\hline $\begin{array}{l}\text { Adding other image } \\
\text { formats to DTF }\end{array}$ & Yes & Yes & Yes & No & Yes \\
\hline $\begin{array}{l}\text { Adding relevant } \\
\text { clinical info to DTF }\end{array}$ & Excellent & Excellent & Excellent & No & Excellent \\
\hline $\begin{array}{l}\text { DTF as reference } \\
\text { library - user-defined } \\
\text { searches }\end{array}$ & Excellent & Excellent & Excellent & Limited & Excellent \\
\hline $\begin{array}{l}\text { DTF as exam } \\
\text { preparation - } \\
\text { 'unknowns' }\end{array}$ & Excellent & Excellent & Excellent & Limited & Excellent \\
\hline Personal use & Limited & Excellent & Excellent & Excellent & Excellent \\
\hline $\begin{array}{l}\text { Institutional/ } \\
\text { practice use }\end{array}$ & Limited & Excellent & Excellent & Limited & Excellent \\
\hline $\begin{array}{l}\text { Multi-institutional } \\
\text { use }\end{array}$ & Excellent & Excellent & Excellent & Limited & No \\
\hline Vendor-independent & Yes & Yes & Yes & No & Yes \\
\hline Software & Web interface & Web interface & Web interface & PACS/RIS interface & Web interface \\
\hline Hardware & Client PCs & Server and client PCs & $\begin{array}{l}\text { Server and client } \\
\text { PCs }\end{array}$ & Client PCs & $\begin{array}{l}\text { Server and client } \\
\text { PCs }\end{array}$ \\
\hline Cost & US\$8 000 & $\begin{array}{l}\text { Free, software must run } \\
\text { on a server }\end{array}$ & US\$8 000 & $\begin{array}{l}\text { Server software and } \\
\text { hardware included in } \\
\text { PACS, free of charge. } \\
\text { Client usage may be } \\
\text { license bound and at } \\
\text { additional cost. }\end{array}$ & US\$8 000 \\
\hline
\end{tabular}

sells for about US\$8 000 and MyPacs.net for a similar amount. MyPacs was originally developed to be an internet-based system but may now be purchased to use as a stand-alone institution-based system.

Several other innovative ways have been described to create DTF systems. One such system uses Advantage Windows workstations (General Electric Medical Systems, Milwaukee, USA) - a tagged image file format (TIFF) file is created from the relevant images and then stored on another computer connected to the local network which supports file transfer protocol (FTP), after which such files are manually entered into a database. This is a reasonable approach but requires programming expertise. $^{11}$

Another way is to export images from workstations to other computers using inexpensive, commercially available 'screen-grab' software. Products such as Snag It (Tech-Smit, East Lansing, MI, USA, www.snagit.com) allow images to be sent directly to a remote server. Although simple in essence, this solution still requires an authoring and database system for the images to be sent to. ${ }^{11}$

An alternative way of transmitting images via email has been described: images and some text are sent via email to the teaching file database. The images and text are automatically extracted from the email message and captured into the database, with only the need to add finishing touches to the case at a later stage.

\section{Internet-based technology}

Internet-based teaching files in essence require DTF cases to be stored on an internet-based server, which then allows access to these 
cases from remote locations. This has the obvious advantage of being accessible from remote, non-work network-related locations (such as from home). However, this raises several issues of concern. Firstly, there is the issue of privacy, with the anonymity of images being essential (especially if accessible over the internet by nearly anyone). The second issue of concern is copyright, with the question of whether the creator of the DTF case or the software developer company holds the copyright. ${ }^{1}$ Thirdly, to utilise such a system, internet connectivity is required; this may not always be possible, depending on bandwidth availability (developing countries) as well as IT regulations of the practice or institution.

Online teaching files fall into two categories: static websites, where cases are stored in hypertext mark-up language (HTML) documents indexed by a list of hyperlinks and, secondly, dynamic (database-driven) web applications.

For static websites, each case is created as an HTML document that contains images and text, with a labelled hyperlink from the collection index page. There are numerous static websites available on the web, a prime example being Eurorad (www.eurorad.org).

Several dynamic database internet-based DTF authoring packages are available for use in developing image libraries. One such is MyPacs (www.mypacs.net), which allows radiologists to create their own teaching files cases from any web browser to add to the large growing collection of cases. ${ }^{16}$ The software supports over 60 file formats and automatically converts such files into the appropriate format used in MyPacs cases. Access to DTF cases can be restricted to the authoring radiologist or, alternatively, be made freely available to all users. Medpix is another free database of images accessible from the internet (rad.usuhs.mil/medpix). ${ }^{14}$ Radiologists can submit cases, but they have to be peer-reviewed by the editorial committee before being accepted. BrighamRad (brighamrad.harvard.edu/), the online teaching file of Brigham and Women's Hospital in Boston, USA, is another online database-driven system. This DTF database is available to external users over the internet, but individuals or institutions not affiliated to Brigham and Women's Hospital may not contribute cases. ${ }^{17}$

\section{Hybrid systems}

These systems were originally developed as intuition-based technologies with the option of connecting to other similar systems via the internet or over a wide area network (WAN). This group has the added advantage of locally based content that can be augmented by content from similar systems not in the same location. These systems then are the ideal configuration where multiple sites require their own DTF library but still want the functionality to connect to other similar sites to broaden their content. Examples of hybrid systems are MIRC (Medical Image Resource Centre) (MIRC, www.mirc.rsna.org) and MyPacs.net (www. mypac.net).

MIRC is an ambitious attempt by the Radiological Society of North America (RSNA) to link online teaching files and construct a global library of digital images and supporting content. MIRC is available as a free program from the RSNA MIRC site. The ultimate aim is to facilitate content searching where a user would only need to log onto one site that searches all available resources, instead of the user needing to search each site individually. This aim will be made possible because every participating image repository files its index card with MIRC. It is however also possible to restrict access to certain files, if needed. MIRC makes use of the extendible mark-up language (XML), a so-called metalanguage that can be used to pass information between different computer systems that would otherwise be unable to communicate. Any MIRC site functions as both a query service and a storage service. In its query service role, it provides access to the entire MIRC (if connected via the internet, or only to the local cases if not) by presenting a query form to the user, distributing the search criteria to all selected storage services, collating the responses and presenting them back to the user. In its storage service role, it responds to the query received from the query service, searches its index for documents meeting the search criteria, and returns abstracts and links to those documents to the query service. ${ }^{1,2,18}$ Several add-ons for the MIRC software have been developed, aiding with MIRC integration in PACS systems among others.

\section{Requirements of DTF at a tertiary institution in South Africa}

Very little information is available on the specific requirements of a DTF system at tertiary institutions in South Africa. Searches on PubMed (www.pubmed.com), Medline and Google (www.google.com) on this subject yielded no suitable literature on the specific subject of requirements at tertiary institutions in South Africa.

Requirements set in this regard at the Pretoria Academic Hospital in South Africa during implementation of the current DTF system related to ease of use, the ability to host multiple users, the option of DTF case confirmation before publishing, and cost of implementating such a system.

Another requirement - that of compatibility with other systems in the possibility of a national DTF archive - is currently in the planning stage (initiated as a joint effort between Pretoria Academic Hospital and Tygerberg Hospital).

\section{The road forward}

In the recent past, radiology as a whole has been transformed with the advent of digital systems. There have been significant changes, not only in the digital acquisition of images but also in the digitisation of image review, image manipulation, image reporting and distribution of reports to the referring clinicians. The advent of digital radiology with the implementation of RIS/PACS systems has changed radiology forever. Case libraries for teaching unfortunately did not receive the same jolt in advancement. However, DTFs will become the standard method of creating, archiving and displaying relevant cases for teaching purposes as the radiological field gradually progresses into digital radiology.

On a general scale, implementation of DTF library systems in the RIS/PACS environment should be actively pursued by vendors, with the focus on implementing an integrated solution. If possible, these solutions should be cross-vendor compatible to facilitate DTF library duplication or joint DTF library ventures between institutions operating software from different vendors. The logical choice in this instance would be to build on the existing IHE TCE protocol and to ensure that vendor solutions conform to and are fully compliant with the IHE TCE protocol. If third-party software is utilised, this should also conform to and be compliant with the IHE TCE protocol. 
On a national scale in South Africa, the focus should be on developing local DTF libraries at the major academic and tertiary institutions. Once these are implemented, a national DTF library should be the goal, either by local DTF library duplication and exchange with other institutions, or possibly even a centralised DTF library accessible from all sites. This can, however, only be achieved if there is compatibility between the systems implemented at the different institutions.

Another recently proposed application of the DTF library software or other form of electronic format is the use of such cases during examinations for postgraduate degree purposes. However, this brings with it some challenges, including (but not limited to) logistical problems, ensuring proper image quality during display (especially if projected), and the introduction of a new medium in established examinations. This form of examination of postgraduate candidates will most likely require some further research and development before it will become standard practice.

\section{Conclusion}

Radiology teaching files are an important and fundamental aspect of radiology training. The advent of digital radiology, RIS/PACS and the resulting transformation of modern radiology departments into a digital environment have led to the traditional teaching file system rapidly becoming obsolete. With these changes, the DTF system has stepped to the forefront and will most likely be the manner in which teaching file cases will be submitted, organised and archived.

Implementation of a DTF system, especially in radiology departments equipped with a RIS/PACS system, is essential. Unfortunately, development of vendor-specific DTF solutions has lagged behind the advances seen in the other fields of digital radiology.

There are several different types of DTF solutions, including thirdparty applications, each with its own advantages and disadvantages. These should be carefully reviewed before implementation.
Academic and tertiary institutions, especially in South Africa, will require specific needs for a DTF system which will be different from international first-world institutions or private sector practices (whether national or international). The cost of system implementation, among other factors, is a major consideration in the South African context.

1. Scarsbrook AF, Foley PT, Perris RW, et al. Radiological digital teaching file development: an overview. Clinical Radiology 2005;60:831-837.

2. Monkolwat P, Bhalodia P, Makori A, et al. Integrating MIRC-compliant semi-automated teaching files into PACS work flow. Radiographics 2005;25:543-548.

3. Rosset $\mathrm{A}$, Ratib $\mathrm{O}$, Geissbuhler $\mathrm{A}$, et al. Integration of a multi-media teaching and reference database in a PACS environment. Radiographics 2002;22:1567-1577.

4. Wiggins RH, Davidson H, Dilda P, et al. The evolution of filmless radiology teaching. J Digit Imaging 2001;14:236-237.

5. Tran TH, Roach NA, O'Kane PL, Thune M. Creating a digital radiographic teaching file and database using a PC and common software. Am J Roentgenol 2000;175:325-327.

6. Wangel M, Niemitukia L, Katia T, et al. WWW: an effective way of teaching radiology. Comput Methods Programs Biomed 2001;66:91-98.

7. Maleck M, Fischer M, Kammer B, et al. Do computers teach better? A media comparison study for casebased teaching in radiology. RadioGraphics 2001;21:1025-1032.

8. Goldberg D, DeMarco JK, Parikh T. Internet based interactive teaching file for neuroradiology. Am J Roentgenol 2000;175:1371-1373.

9. Mehta A, Dreyer KJ, Montgomery M, et al. A World Wide Web Internet engine for collaborative entry and peer review of radiologic teaching files. Am J Roentgenol 1999;172:893-896.

10. Henderson B, Camorlinga S, DeGagne JC. A cost-effective web-based teaching file system. J Digit Imaging 2004;17:87-91.

11. Maldjian JA, Listerud J. Automated teaching file and slide database for digital images. Am J Roentgenol 2000;175:1249-1251.

12. Halsted MJ, Moskovitz J, Johnson N, et al. A simple method of capturing PACS and other radiographic images for digital teaching files or other image repositories. Am J Roentgenol 2002;178:817-819.

13. Jakobovits R. MyPACS teaching file management system. http://www.mypacs.net (accessed 1 March 2008).

14. Smirniotopoulos JG. MedPix" medical image database. http://rad.usuhs.mil/medpix (accessed 1 March 2008).

15. Rosset A. CASIM@GE teaching file database. http://pubimage.hcuge.ch (accessed 1 March 2008).

16. Weinberger E, Jakobovits R, Halsted M. MyPACS.net: a webbased teaching file authoring tool. Am J Roentgenol 2002;179:579-582.

17. Holman BL, Mammone GL, Greenes RA, et al. Brighamrad: An approach to education, decision support, information dissemination, and collaboration via the World Wide Web. http://ej.rsna.org/EJ_0_96/001096/0010-96.sit/title_page.html RSNA EJ 1996 (accessed 1 March 2008).

18. Medical Imaging Resource Center. Mircwiki.rsna.org. (accessed 1 March 2008). 